\title{
EQUIVALENCE OF FAMILIES OF FUNCTIONS ON THE NATURAL NUMBERS
}

\author{
CLAUDE LAFLAMME
}

\begin{abstract}
We present some consequences of the inequality $\mathfrak{u}<\mathfrak{g}$ among cardinal invariants of the continuum, which has previously been shown to be consistent relative to ZFC. We are interested in its effect on two orderings of families of functions on the natural numbers; in particular we show that, under $\mathfrak{u}<\mathfrak{g}$, there are exactly five equivalence classes for both orderings (excluding the families bounded by a fixed constant function). This implies, under the same hypothesis, the existence of exactly four classes of rarefaction of measure zero sets.
\end{abstract}

\section{INTRODUCTION}

The primary purpose of this paper is to present some consequences of the inequality $\mathfrak{u}<\mathfrak{g}$ among cardinal invariants of the continuum, which was shown to be consistent relative to ZFC in [3]. We are interested in its effect on two orderings of families of nondecreasing functions on the natural numbers. The first ordering has been defined by Göbel and Wald in [4] for growth types, which are special families of functions, to study slenderness classes of Abelian groups. They showed in particular that there are at least four equivalence classes of growth types, and it was proved in [3] that there are exactly four classes if one assumes $\mathfrak{u}<\mathfrak{g}$. A second ordering was defined in [6] on families of functions canonically obtained from the covers of measure zero sets in order to study their so-called rarefaction (following Borel). It was proved again that there are at least four equivalence classes of such families of functions under this ordering, although only three were actually equivalence classes of growth types; the question whether this minimum number could be attained remained open.

It seemed natural to study both orderings on arbitrary families of (nondecreasing) functions on the natural numbers and the effect of $\mathfrak{u}<\mathfrak{g}$ on the induced ordering of the equivalence classes. Excluding the families bounded by a fixed constant function, the minimum number is five for both orderings. It is the main result of this paper that there are exactly five (linearly ordered) classes under $\mathfrak{u}<\mathfrak{g}$; the second highest class never contains a growth type and the lowest one never arises from a measure zero set. In particular, under $\mathfrak{u}<\mathfrak{g}$,

Received by the editors February 9, 1989 and, in revised form, December 28, 1989.

1980 Mathematics Subject Classification (1985 Revision). Primary 03E35; Secondary 28A05, 04A20.

Supported by an FCAR Postdoctoral Research Fellowship from the Province of Quebec. 
there are exactly four equivalence classes of rarefaction of measure zero sets, which answers a question of [6].

A curious consequence of this minimal number five being attained, is the following: given any nonempty family $\mathscr{A}$ of infinite sets, there is a finite-toone function $f$ such that either $f(\mathscr{A})$ is dense in $\left\langle[\omega]^{\omega} ; \supseteq^{*}\right\rangle\left(A \subseteq^{*} B\right.$ means that $A \backslash B$ is finite), or $f(\mathscr{A})$ generates an ultrafilter, or else $f(\mathscr{A})$ consists only of cofinite sets. This implies in particular the principle NCF of [1].

\section{NOTATION AND PRELIMINARIES}

We write $\omega$ for the set of natural numbers, and $\omega \nearrow \omega$ for the set of nondecreasing functions on $\omega$. We shall often use $f \leq g$ to abbreviate $(\forall n) f(n) \leq g(n)$, and $f \leq^{*} g$ for $\left(\forall^{\infty} n\right) f(n) \leq g(n)$; here " $\forall{ }^{\infty} n$ " means "for all but finitely many $n$ " and similarly " $\exists^{\infty} n$ " means "there exists infinitely many $n$." A function $f$ is called finite-to-one if $f^{-1}\{n\}$ is finite for each $n$. We use $\mathscr{X}, \mathscr{Y}$ to denote families of functions. The infinite subsets of $\omega$ are denoted by $[\omega]^{\omega}$ and we use $\mathscr{A}, \mathscr{B}$ to denote families of such sets. We define $\mathscr{Z}(\mathscr{A})=\{f \in \omega \nearrow \omega:\{n: f(n) \leq n\} \in \mathscr{A}\}$, and hence each $f \in \mathscr{Z}(\mathscr{A})$ is $\leq$ to a function of the form $\operatorname{next}(A,-)$ for some $A \in \mathscr{A}$, where $\operatorname{next}(A, n)=$ the next element of $A$ greater than or equal to $n$.

An ultrafilter is a proper family of infinite sets closed under finite intersections, supersets, and maximal with respect to those properties; in particular it must contain $X$ or $\omega \backslash X$ for any $X \subseteq \omega$, and must be nonprincipal. We use $\mathscr{U}, \mathscr{V}$ to denote ultrafilters. The letter $\mathfrak{u}$ will be used to denote the least cardinality of a family of sets generating an ultrafilter.

We now recall the definition in [3] of the cardinal $\mathfrak{g}$. A family of infinite sets $\mathscr{S}$ is called groupwise dense if:

1. $Y^{\prime} \in \mathscr{S}$ whenever $Y^{\prime} \subseteq^{*} Y$ for some $Y \in \mathscr{S}$.

2. For every infinite family $\pi$ of disjoint finite subsets of $\omega$, there exists a (necessarily infinite) subfamily of $\pi$ whose union is a member of $\mathscr{S}$.

It is observed in [3] that in 2, it suffices to require $\pi$ to be a partition of $\omega$ into intervals.

Then $\mathfrak{g}$ is defined as the least cardinality of a collection of groupwise dense families with empty intersection. The inequality $\mathfrak{u}<\mathfrak{g}$ was shown in [3] to imply the principle of near coherence of filters $(\mathrm{NCF})$, and was verified to hold in various known models of $\mathrm{ZFC}+\mathrm{NCF}$; hence is relatively consistent with ZFC. The reader is referred also to [2] for a review of NCF and $\mathfrak{u}<\mathfrak{g}$.

We say that a function is bounded if it is bounded by a constant function; we say that a family of functions $\mathscr{X}$ is bounded if

$$
(\exists g \in \omega \nearrow \omega)(\forall f \in \mathscr{X})\left(\forall^{\infty} n\right)[f(n) \leq g(n)] .
$$

$\mathscr{X}$ is said to dominate $\mathscr{Y}$ if

$$
(\forall g \in \mathscr{Y})(\exists f \in \mathscr{X})\left(\forall^{\infty} n\right)[f(n) \geq g(n)] .
$$

$\mathscr{Z}$ is a dominating family if it dominates $\omega \nearrow \omega$.

We will be interested in two orderings of families of functions.

Definition 1. Let $\mathscr{X}, \mathscr{Y}$ be any families of functions.

1. $\mathscr{X} \preceq^{1} \mathscr{Y}$ iff $(\exists r \in \omega \nearrow \omega)(\forall f \in \mathscr{X})(\exists g \in \mathscr{Y})\left(\forall^{\infty} n\right)[r(g(n)) \geq f(n)]$.

2. $\mathscr{X} \preceq^{2} \mathscr{Y}$ iff $(\exists r \in \omega \nearrow \omega)(\forall f \in \mathscr{X})(\exists g \in \mathscr{Y})\left(\forall^{\infty} n\right)[g(r(n)) \geq f(n)]$. 
The ordering $\preceq^{1}$ has been used in [6] to classify measure zero sets with respect to their open coverings; the ordering $\preceq^{2}$ has been introduced in [4] to study slenderness classes of Abelian groups and has been investigated in $[4,5$, 1 and 3].

Since both orderings are concerned with the rapidly growing functions in each family and with no effect on finite modifications of functions, we shall assume throughout that our families of functions $\mathscr{Z}$ are closed downward under $\leq^{*}$; that is $g \in \mathscr{X}$ whenever $g \leq^{*} f$ for some $f \in \mathscr{X}$. In particular, we can replace $\forall^{\infty} n$ by $\forall n$ in Definition 1 above.

\section{THE TOP AND BOTTOM OF THE ORDERINGS}

We are interested in the induced orderings on the equivalence classes of families of functions.

As in [1], we can quickly dispense with the bottom of the orderings. Indeed, let $\mathscr{B}_{k}=\{f:(\forall n) f(n) \leq k\}$, and $\mathscr{B}=\bigcup_{k \in \omega} \mathscr{B}_{k}$. Then $\mathscr{B}_{k} \prec^{2} \mathscr{B}_{k+1} \prec^{2} \mathscr{B}$ and any family $\mathscr{X}$ is either above $\mathscr{B}$ or equivalent to some $\mathscr{B}_{k}$ in the second ordering. Hence the bottom of the ordering $\preceq^{2}$ has type $\omega+1$. On the other hand, $\mathscr{B}_{k+1} \preceq^{1} \mathscr{B}_{k} \prec^{1} \mathscr{B}$ for each $k$ and any family is either above $\mathscr{B}$ or equivalent to some (all) $\mathscr{B}_{k}$; hence the bottom of the $\preceq^{1}$ ordering has type 2 . Because of this slight discrepancy between the two orderings and the fact that the families $\mathscr{B}_{k}$ are of no real interest, we shall only consider families above (and including) $\mathscr{B}$. We shall write $\preceq$ to denote either $\preceq^{1}$ or $\preceq^{2}$; the reason being that, as we shall see, both orderings have much in common.

By our convention, the bottom class now consists of the family $\mathscr{B}$ of bounded functions, and we call it the bounded class, denoted by $\mathscr{B}$. Next consider, as in [1], the family $\mathscr{L}=\left\{f \in \omega \nearrow \omega:\left(\forall^{\infty} n\right) f(n) \leq n\right\}$. Then $\mathscr{L} \preceq \mathscr{Z}$ or $\mathscr{X} \preceq \mathscr{B}$ for any $\mathscr{X}$. Further, $\mathscr{X} \sim \mathscr{L}$ iff $\mathscr{X}$ is a bounded family of functions containing at least one unbounded function. We call the class of such families of functions (containing $\mathscr{L}$ ) the low class, and denote it also by $\mathscr{L}$.

As for the top of the orderings, we certainly have $\mathscr{Z}=\omega \nearrow \omega \succeq \mathscr{Y}$ for any $\mathscr{Y}$; more generally $\mathscr{X} \succeq \mathscr{Y}$ for any $\mathscr{Y}$ if $\mathscr{X}$ is a dominating family. It is not hard to show further that $\mathscr{Z}$ is a dominating family whenever $\mathscr{X} \succeq \omega \nearrow \omega$ and hence the top class in both orderings consists precisely of the dominating families; we call it the dominating class and denote it by $\mathscr{D}$.

Now if $\mathscr{Z}$ is not in the $\mathscr{D}$-class, then $\mathscr{X}$ is not dominating and hence

$$
(\exists g \in \omega \nearrow \omega)(\forall f \in \mathscr{X})\left(\exists^{\infty} n\right)[f(n) \leq g(n)] ;
$$

we conclude that $\mathscr{Z} \subseteq\{f:\{n: f(n) \leq g(n)\}$ is infinite $\}$. This suggests the study of the family $\mathscr{X}\left([\omega]^{\omega}\right)$. Indeed, $\mathscr{X}\left([\omega]^{\omega}\right) \prec \mathscr{D}$ since it is not a dominating family, and $\mathscr{X}\left([\omega]^{\omega}\right) \succ \mathscr{L}$ since it is not a bounded family. We shall see that it is actually in the second highest class, that we call the high class, denoted by $\mathscr{H}$.

Definition 2. A family $\mathscr{X}$ has the $\mathscr{H}$-property if for some unbounded $g \in$ $\omega \nearrow \omega, \mathscr{X} \supseteq\{f:\{n: f(n) \leq g(n)\}$ is infinite $\}$.

The next two propositions are proved in [6] for the first ordering.

Proposition 3. If $\mathscr{Z}$ has the $\mathscr{H}$-property and $\mathscr{Y}$ is not dominating, then $\mathscr{X} \succeq \mathscr{Y}$. 
Proof. We first supply the proof for the $\preceq^{2}$ order.

By hypothesis, we can fix a function $h$ and an unbounded function $g$ such that

$$
(\forall f \in \mathscr{Y})[f(n) \leq h(n) \text { for infinitely many } n]
$$

and

$$
\mathscr{Z} \supseteq\{\bar{g}: \bar{g}(n) \leq g(n) \text { for infinitely many } n\} .
$$

We need to find $r \in \omega \nearrow \omega$ such that

$$
(\forall f \in \mathscr{Y})(\exists \bar{g} \in \mathscr{X})\left(\forall^{\infty} n\right)[\bar{g}(r(n)) \geq f(n)] .
$$

Choose $r$ strictly increasing such that $g(r(n)) \geq h(n)$ for each $n$. We claim that $r$ works. Fix $f \in \mathscr{Y}$ and pick an increasing sequence $\left\langle n_{i}: i \in \omega\right\rangle$ such that $f\left(n_{i}\right) \leq h\left(n_{i}\right)$ for each $i$. Define $\bar{g}(n)=g\left(r\left(n_{i+1}\right)\right)$ if $n \in\left(r\left(n_{i}\right), r\left(n_{i+1}\right)\right]$. Then $\bar{g} \in \mathscr{X}$ since $\bar{g}\left(r\left(n_{i}\right)\right)=g\left(r\left(n_{i}\right)\right)$ for each $i$. But now, for each $n$, say $n \in\left(n_{i}, n_{i+1}\right]$,

$$
\bar{g}(r(n))=g\left(r\left(n_{i+1}\right)\right) \geq h\left(n_{i+1}\right) \geq f\left(n_{i+1}\right) \geq f(n)
$$

as desired.

The proof for the $\preceq^{1}$ order (from Lemma 3.4 of [6]) is obtained as above by choosing $r$ such that $r(g(n)) \geq h(n)$ for each $n$ and by using $\bar{g}(n)=g\left(n_{i+1}\right)$ for $n \in\left(n_{i}, n_{i+1}\right]$.

The following shows that having the $\mathscr{H}$-property without being dominating characterizes the $\mathscr{H}$-class.

Proposition 4. If $\mathscr{Y}$ has the $\mathscr{H}$-property and $\mathscr{X} \succeq \mathscr{Y}$, then $\mathscr{X}$ has the $\mathscr{H}$ property as well.

Proof. We first supply the proof for the $\preceq^{2}$ order.

By hypothesis, we can fix an unbounded function $g$ such that

$$
\mathscr{Y} \supseteq\{f: f(n) \leq g(n) \text { infinitely often }\}
$$

and $r$, without loss of generality strictly increasing, such that

$$
(\forall f \in \mathscr{Y})(\exists h \in \mathscr{X})(\forall n)[h(r(n)) \geq f(n)] .
$$

Define $\bar{g}(n)=g(m-1)$ if $n \in[r(m), r(m+1))$. Then $\bar{g}$ is unbounded and we claim that it witnesses the $\mathscr{H}$-property of $\mathscr{X}$. Indeed, suppose $h(n) \leq \bar{g}(n)$ for infinitely many $n$, then $h(r(m+1)) \leq g(m)$ for infinitely many $m$, and hence the function $f(m)=h(r(m+1)) \in \mathscr{Y}$. Since $\mathscr{X} \succeq \mathscr{Y}$, we can find some $\bar{h} \in \mathscr{X}$ such that

$$
\bar{h}(r(n)) \geq f(n)=h(r(n+1)) \text { for each } n
$$

and we conclude that, for each $n$, say $n \in[r(m), r(m+1))$,

$$
\bar{h}(n) \geq \bar{h}(r(m)) \geq h(r(m+1)) \geq h(n) .
$$

Hence $h \in \mathscr{X}$ as desired since it closed downward.

The proof for the $\preceq^{1}$ order (from Lemma 3.5 of [6]) is obtained as above by defining $\bar{g}(n)=m$ if $r(m) \leq g(n)<r(m+1)$ (so $r(\bar{g}(n)) \leq g(n)$ for each $n)$.

At this point, we have covered all Borel (even analytic) families of functions (with respect to the topology induced by the product topology on the product ${ }^{\omega} \omega$ of countably many copies of $\omega$, each copy having the discrete topology). Indeed, we can modify the argument of [1] about a similar result on ideals. 
Proposition 5. If $\mathscr{X}$ is an analytic family of functions on $\omega$, then either $\mathscr{X}$ is bounded, or $\mathscr{Z}$ has the $\mathscr{H}$-property.

Proof. A tree $\mathscr{T} \subseteq{ }^{<\omega} \omega$ is called superperfect if any $s \in \mathscr{T}$ has an extension $t \in \mathscr{T}$ with infinitely many immediate successors.

The proof relies (as in [1]) on the results of [7 and 8] that such an $\mathscr{X}$ must either be included in a $K_{\sigma}$-set (that is included in a countable union of compact sets), which means precisely that it is bounded, or else $\mathscr{T}_{\mathscr{L}}=\left\{\chi_{X} \mid n: X \in\right.$ $\mathscr{Z}, n \in \omega\}$ must contain a superperfect subtree $\mathscr{T}$, where $\chi_{X} \in{ }^{\omega} \omega$ is the function enumerating $X$ in increasing order. It thus suffices to show that $\mathscr{X}$ has the $\mathscr{H}$-property whenever $\mathscr{T}_{\mathscr{L}}$ contains a superperfect subtree $\mathscr{T}$.

By pruning $\mathscr{T}$, we can assume that each branching node is infinitely branching, and further that whenever $s^{\wedge}\langle n\rangle \in \mathscr{T}$, where $s$ is a branching node, then $n>$ range $(s)$. Now define

$$
\begin{aligned}
\bar{g}(n)= & \text { the least } m, \\
& \text { such that for all branching nodes } s \in \mathscr{T} \cap^{<\omega}(n+1), \\
& \text { if } k \text { is the least number greater than } n \text { such that } s^{\wedge}\langle k\rangle \in \mathscr{T}, \\
& \text { then } s^{\wedge}\langle k\rangle \text { has a branching extension in } \mathscr{T} \cap{ }^{\leq m} \omega .
\end{aligned}
$$

Then $\bar{g}$ is well defined since $\mathscr{T} \cap^{<\omega} n$ is finite for each $n$ by our hypothesis on $\mathscr{T}$. Now put $g(m)=$ the largest $n$ such that $\bar{g}(n) \leq m$. Hence $\bar{g}(g(m)) \leq m$ by definition. We claim that $g$ witnesses the $\mathscr{H}$-property of $\mathscr{X}$. Indeed, $g$ is unbounded, and given $h$ such that $h(n) \leq g(n)$ for infinitely many $n$, we must prove that $h$ belongs to $\mathscr{Z}$. It suffices to produce an $f \in \mathscr{X}$ which eventually majorizes $h$, and we do this by induction. Let $r \in{ }^{m_{0}} \omega$ be the root of $\mathscr{T}$, and put $f \mid m_{0}=r$. Now assume that $s=f \mid m_{i} \in \mathscr{T}$ has been defined, and is a branching node of $\mathscr{T}$. Choose $n>m_{i}$ such that $g(n) \geq h(n)$, range $\left(f \mid m_{i}\right)$. Let $k$ be the least integer greater than $g(n)$ such that $s^{\wedge}\langle k\rangle \in \mathscr{T}$. Since $\bar{g}(g(n)) \leq n$, we can find a branching extension $t \in$ $m_{i+1} \omega \cap \mathscr{T}$ of $s^{\wedge}\langle k\rangle$ with $m_{i+1} \leq n$. Define $f \mid m_{i+1}=t$. Observe that if $j \in\left[m_{i}, m_{i+1}\right)$, then $f(j)=t(j) \geq t\left(m_{i}\right)=k \geq g(n) \geq h(n) \geq h\left(m_{i+1}\right) \geq h(j)$. This completes the construction and the proof of the proposition.

\section{THE MIDDLE CLASS(ES)}

For a class to lie between the low class and the high class, we must search for a family in between $\mathscr{X}$ (cofinite sets) which is in $\mathscr{L}$ and $\mathscr{Z}\left([\omega]^{\omega}\right)$ which is in $\mathscr{H}$. It seems natural to look at $\mathscr{Z}(\mathscr{U})$ for an ultrafilter $\mathscr{U}$. Indeed, one easily shows that such a family is unbounded (hence above $\mathscr{L}$ ), and does not have the $\mathscr{H}$-property (hence below $\mathscr{H}$ ); it must consequently constitute a new class which we call the $\mathscr{U}$-class. The two orderings restricted to these families are inverse of each other as the following shows.

Lemma 6. For any two ultrafilters $\mathscr{U}, \mathscr{V}$, we have

$$
\mathscr{X}(\mathscr{U}) \preceq^{1} \mathscr{X}(\mathscr{V}) \text { iff } \mathscr{X}(\mathscr{V}) \preceq^{2} \mathscr{X}(\mathscr{U}) .
$$

Proof. (Compare with the proof of Lemma 5.4 of [1].) Assume that $\mathscr{Z}(\mathscr{U}) \preceq^{1}$ $\mathscr{X}(\mathscr{V})$, and hence by definition we can fix $r$ strictly increasing such that

$$
(\forall A \in \mathscr{U})(\forall B \in \mathscr{V})(\forall n)[\operatorname{next}(A, n) \leq r(\operatorname{next}(B, n))=\operatorname{next}(r(B), r(n))] .
$$


This is equivalent to

$$
(\forall A \in \mathscr{U})(\exists B \in \mathscr{V})\left[r(B) \text { is disjoint from } \bigcup_{n}[r(n), \operatorname{next}(A, n))\right] .
$$

But, as $\mathscr{V}$ is an ultrafilter, this is equivalent to

$$
(\forall A \in \mathscr{U})(\forall B \in \mathscr{V})(\exists b \in B)\left[r(b) \notin \bigcup_{n}[r(n), \operatorname{next}(A, n))\right] .
$$

Fix $A$ and $B$ for a moment and let $a$ be the largest element of $A$ which is $\leq r(b)$. If $n \leq a$, then $r(b) \notin[r(n), \operatorname{next}(A, n))$ as $\operatorname{next}(A, n) \leq a \leq r(b)$. Hence, as $r$ is nondecreasing and $r(b)<\operatorname{next}(A, a+1)$, to say that $r(b) \notin$ $\bigcup_{n}[r(n), \operatorname{next}(A, n))$ is equivalent to

$$
a \leq r(b)<r(a+1) .
$$

Defining $s(n)=r(n)+1$, the last line becomes equivalent to $b \leq a<s(b)$, since $r$ and $s$ are strictly increasing. So we deduce that $\mathscr{X}(\mathscr{U}) \preceq^{1} \mathscr{X}(\mathscr{V})$ if and only if there is a strictly increasing $s$ such that

$$
(\forall B \in \mathscr{V})(\forall A \in \mathscr{U})(\exists b \in B)(\exists a \in A)[b \leq a<s(b)] .
$$

Going now backward, this is equivalent to

$$
(\forall B \in \mathscr{V})(\forall A \in \mathscr{U})(\exists a \in A)\left[a \notin \bigcup_{n}[s(n), \operatorname{next}(B, n))\right]
$$

and hence equivalent to

$$
(\forall B \in \mathscr{V})(\exists A \in \mathscr{U})\left[A \text { is disjoint from } \bigcup_{n}[s(n), \operatorname{next}(B, n))\right],
$$

but the statement in square brackets is equivalent to

$$
(\forall n)[\operatorname{next}(B, n) \leq \operatorname{next}(A, s(n))]
$$

and this is precisely $\mathscr{X}(\mathscr{V}) \preceq^{2} \mathscr{X}(\mathscr{U})$ as desired.

A description of the ordering $\preceq^{2}$ with respect to the families of the form $\mathscr{X}(\mathscr{U})$ has been given in [1] and hence a similar (inverted) description applies to the first ordering. In particular, under Martin's Axiom, there are $2^{2^{\mathrm{N}_{0}}}$ ultrafilters producing pairwise incomparable families, each lying in a chain of size $2^{2^{\mathrm{N}_{0}}}$. The two orderings are thus different in general; however an exact relationship between both orderings in ZFC is unknown (for example, are they isomorphic?)

However, the goal of this paper is to show the effect of $\mathfrak{u}<\mathfrak{g}$ on these orderings.

Theorem 7. Under $\mathfrak{u}<\mathfrak{g}$, there are exactly five classes of families of functions (for either ordering).

Recall that we excluded families bounded by a fixed constant function, whose orderings have been described in $\S 3$.

The proof will be divided in two parts. 
Proposition 8. Assume $\mathfrak{u}<\mathfrak{g}$. If a family $\mathscr{Z}$ does not have the $\mathscr{H}$-property then it is dominated by a set of functions of size $\mathfrak{u}$.

Proof. Recall that the statement that $\mathscr{Z}$ does not have the $\mathscr{H}$-property means that

$$
(\forall \text { unbounded } f)(\exists g)\left[\left(\exists^{\infty} n\right) g(n) \leq f(n) \text { and } g \notin \mathscr{X}\right],
$$

and $g \notin \mathscr{X}$ means that for all $h \in \mathscr{X}, h(n)<g(n)$ infinitely often since $\mathscr{X}$ is assumed to be closed downward.

Fix an ultrafilter $\mathscr{U}$ generated by $\mathfrak{u}$ sets $\left\langle X_{\alpha}: \alpha<\mathfrak{u}\right\rangle$. For each $\alpha$, define

$$
\begin{aligned}
& \mathscr{S}_{\alpha}=\left\{Y:(\forall h \in \mathscr{X})\left(\exists^{\infty} y \in Y\right)\right. \\
& {\left.\left[h(y) \leq \operatorname{next}(Y, y+1) \text { and } X_{\alpha} \cap[y, \operatorname{next}(Y, y+1)) \neq \varnothing\right]\right\} . }
\end{aligned}
$$

We first claim that each $\mathscr{S}_{\alpha}$ is groupwise dense. Clearly $Y \in \mathscr{S}_{\alpha}$ whenever $Y \subseteq^{*} Y^{\prime}$ for some $Y^{\prime} \in \mathscr{S}_{\alpha}$. We further need to verify that given a partition $\left[\pi_{i}, \pi_{i+1}\right)$ of $\omega$ into intervals, there is an infinite union of them in $\mathscr{S}_{\alpha}$.

By merging intervals, we may assume without loss of generality that $X_{\alpha}$ meets each interval. Define a function $f$ by $f(n)=\pi_{i-1}$ whenever $n \in\left[\pi_{i}, \pi_{i+1}\right)$. By assumption, there is $g \notin \mathscr{X}$ such that $g(n) \leq f(n)$ for infinitely many $n$. Put $X=\{n: g(n) \leq f(n)\}$; we can assume that $g(n)=f(\operatorname{next}(X, n))$, since this function majorizes the old $g$. Since $g \notin \mathscr{X}$, any $h \in \mathscr{Z}$ must satisfy $h(n)<g(n)$ for infinitely many $n$; and for each such $n$ and $x$ the last element of $X$ before $n$,

$$
h(x) \leq h(n)<g(n)=f(\operatorname{next}(X, n)) \leq f(\operatorname{next}(X, x+1)) .
$$

Hence we have a set $X$, such that for all $h \in \mathscr{X}$,

$$
\left(\exists^{\infty} x \in X\right) h(x)<f(\operatorname{next}(X, x+1)) .
$$

We may assume further that $X$ meets each interval $\left[\pi_{i}, \pi_{i+2}\right)$ at most once since any infinite $X^{\prime} \subseteq X$ would have the same property. So we define

$$
Y=\bigcup\left\{\left[\pi_{i}, \pi_{i+1}\right): X \text { meets }\left[\pi_{i+1}, \pi_{i+2}\right)\right\} .
$$

$Y$ is an infinite union since $X$ is infinite. We show that $Y \in \mathscr{S}_{\alpha}$.

Fix $h \in \mathscr{Z}$, and hence $h(x) \leq f(\operatorname{next}(X, x+1))$ for infinitely many $x \in X$. Fix such an $x$, say $x \in\left[\pi_{i+1}, \pi_{i+2}\right)$, then $y=\pi_{i+1}-1 \in Y$ by definition of $Y$; and if $x^{\prime}=\operatorname{next}(X, x+1)$, say $x^{\prime} \in\left[\pi_{j+1}, \pi_{j+2}\right)$, then $j+1 \geq i+3$ by construction. Hence,

$$
f\left(x^{\prime}\right)=\pi_{j}=\operatorname{next}(Y, y+1)
$$

by definition, and we get

$$
h(y) \leq h(x) \leq f(\operatorname{next}(X, x+1))=f\left(x^{\prime}\right)=\operatorname{next}(Y, y+1) .
$$

Also,

$$
y<\pi_{i+1}<\pi_{i+2} \leq \pi_{j}=\operatorname{next}(Y, y+1),
$$

and hence $X_{\alpha}$ meets $[y, \operatorname{next}(Y, y+1))$. Because this holds for infinitely many $y \in Y$, we conclude that $Y \in \mathscr{S}_{\alpha}$ and this shows that $\mathscr{S}_{\alpha}$ is groupwise dense.

Since we are assuming $\mathfrak{u}<\mathfrak{g}$, we can fix $Y \in \bigcap_{\alpha<u} \mathscr{S}_{\alpha}$, and define the function $f_{Y}(n)=|Y \cap(n+1)|$ (so $f_{Y}$ is constant on the intervals determined by $Y$, and $\min f^{-1}\{n\} \in Y$ for each $\left.n\right)$. 
Fix $h \in \mathscr{X}$, and let $Z=\{y \in Y: h(y) \leq \operatorname{next}(Y, y+1)\}$. We now claim that $f_{Y}(Z)$ is in $f_{Y}(\mathscr{U})$. For this, it suffices to verify that $f_{Y}(Z)$ meets each $f_{Y}\left(X_{\alpha}\right)$ for $\alpha<\mathfrak{u}$. But since $Y \in \mathscr{S}_{\alpha}$,

$$
\left(\exists^{\infty} y \in Y\right)\left[y \in Z \text { and } X_{\alpha} \cap[y, \operatorname{next}(Y, y+1)) \neq \varnothing\right],
$$

and hence $f_{Y}(y)=f_{Y}(x)$ if $x \in X_{\alpha} \cap[y, \operatorname{next}(Y, y+1))$.

Finally, since $f_{Y}(\mathscr{U})$ is generated by the sets $\left\langle f_{Y}\left(X_{\alpha}\right): \alpha<u\right\rangle$, there must be an $\alpha$ such that $f_{Y}\left(X_{\alpha}\right) \subseteq f_{Y}(Z)$. Hence, for all $n$,

$$
\begin{aligned}
h(n) & \leq h(\operatorname{next}(Z, n)) \\
& \leq \operatorname{next}(Y, \operatorname{next}(Z, n)+1) \\
& \leq \operatorname{next}\left(Y, \max f^{-1}\left\{\operatorname{next}\left(f_{Y}\left(X_{\alpha}\right), f_{Y}(n)+1\right)\right\}\right) \\
& \leq \min f_{Y}^{-1}\left\{\operatorname{next}\left(f_{Y}\left(X_{\alpha}\right), f_{Y}(n)+1\right)+1\right\} .
\end{aligned}
$$

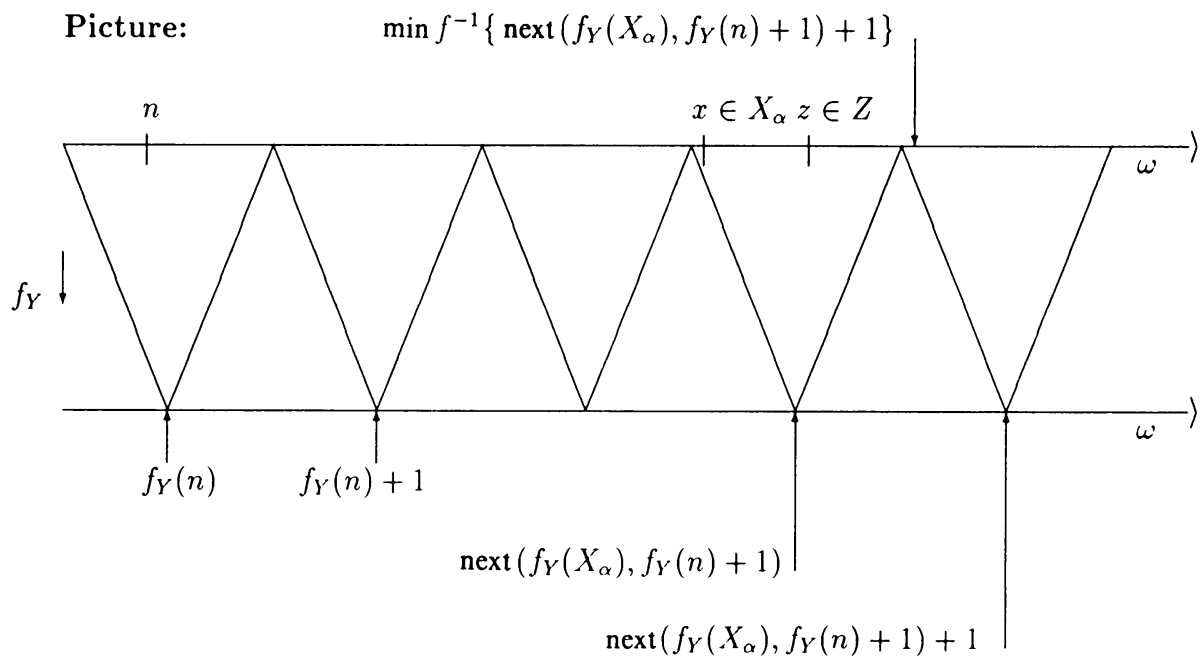

Since $Y$ is fixed and $h$ was an arbitrary element of $\mathscr{X}, \mathscr{Z}$ is dominated by the $\mathfrak{u}$ functions of the form on the last line, and hence the proof is complete.

To complete the proof of the theorem, we use the following lemma whose proof for the first ordering can be found in Lemma 6.3 of [6], and in the proof of $(0) \Rightarrow(1)$ from [3] for the second ordering; they are included here for completeness.

Lemma 9. If $\mathscr{X}$ is an unbounded family and $\mathscr{Y}$ is dominated by a family of functions of size less than $\mathfrak{g}$, then $\mathscr{X} \succeq \mathscr{Y}$.

Proof. We first supply the proof for the $\preceq^{1}$ order. Fix a family $\left\{h_{\alpha}: \alpha<\right.$ $\kappa\}, \kappa<\mathfrak{g}$, dominating $\mathscr{Y}$. For each $\alpha$, define:

$$
\mathscr{A}_{\alpha}=\left\{A \in[\omega]^{\omega}:(\exists f \in \mathscr{X})\left(\forall^{\infty} n\right)\left[\operatorname{next}(A, f(n)) \geq h_{\alpha}(n)\right]\right\} .
$$

We first claim that $\mathscr{A}_{\alpha}$ is groupwise dense. Since it is clearly closed under subsets and finite modifications, it suffices to fix a partition of $\omega$ into intervals $\left[\pi_{i}, \pi_{i+1}\right)$ and show that $\mathscr{A}_{\alpha}$ contains an infinite union of them.

By first merging intervals together if necessary, we may assume without loss of generality that $h_{\alpha}\left(\pi_{i}\right)<\pi_{i+1}$ for each $i$. Now fix an arbitrary function 
$g \in \omega \nearrow \omega$ such that $g\left(\pi_{i}\right) \geq \pi_{i+4}$ for each $i$; since $\mathscr{X}$ is assumed to be unbounded, we can find $f \in \mathscr{X}$ such that $f(n) \geq g(n)$ for infinitely many $n$.

Let $A=\bigcup\left\{\left[\pi_{i+2}, \pi_{i+3}\right):\left(\exists n \in\left[\pi_{i}, \pi_{i+1}\right)\right) f(n) \geq g(n)\right\}$. We show that $A \in$ $\mathscr{A}_{\alpha}$, i.e. that $\operatorname{next}(A, f(m)) \geq h_{\alpha}(m)$ for each $m$. Fix $m$, say $m \in\left[\pi_{i}, \pi_{i+1}\right)$ and let $k=\operatorname{next}(A, f(m))$, say $k \in\left[\pi_{j+2}, \pi_{j+3}\right)$. By definition of $A$, there is $n \in\left[\pi_{j}, \pi_{j+1}\right)$ such that $f(n) \geq g(n)$; hence $f(m) \leq k<\pi_{j+4} \leq g\left(\pi_{j}\right) \leq$ $g(n) \leq f(n) \leq f(k)$. We conclude that $m \leq k$, and therefore $i \leq j+2$. If actually $j \geq i$, then we get $k \geq \pi_{i+2} \geq h_{\alpha}(m)$ and we are done. If on the other hand $j<i$, then again with the $n$ above, we get

$$
k \geq f(m) \geq f(n) \geq g(n) \geq \pi_{j+4} \geq \pi_{i+2} \geq h_{\alpha}(m),
$$

and this completes the proof of the claim.

Since $\kappa<\mathfrak{g}$, we can find $A \in \bigcap_{\alpha<\kappa} \mathscr{A}_{\alpha}$ and define $r(n)=\operatorname{next}(A, n)$; we finally verify that $r$ witnesses $\mathscr{Y} \preceq^{1} \mathscr{X}$. Given $h \in \mathscr{Y}$, choose $\alpha$ such that $h \leq^{*} h_{\alpha} ;$ since $A \in \mathscr{A}_{\alpha}$, there is an $f \in \mathscr{X}$ such that

$$
\left(\forall^{\infty} n\right) r(f(n))=\operatorname{next}(A, f(n)) \geq h_{\alpha}(n) \geq h(n)
$$

as desired.

Now the proof for the $\preceq^{2}$ order. For each $\alpha<\kappa$, define

$$
\mathscr{B}_{\alpha}=\left\{B \in[\omega]^{\omega}:(\exists f \in \mathscr{X})\left(\forall^{\infty} n\right)\left[f(\operatorname{next}(A, n)) \geq h_{\alpha}(n)\right]\right\} .
$$

As above, any $B \in \bigcap_{\alpha<\kappa} \mathscr{B}_{\alpha}$ will yield the desired $r(n)=\operatorname{next}(B, n)$ witnessing $\mathscr{Y} \preceq^{2} \mathscr{Z}$. So we need only verify that each $\mathscr{B}_{\alpha}$ is groupwise dense. But this is also very similar to the above; use any $g$ such that $g\left(\pi_{i}\right) \geq \pi_{i+3}$, pick $f \in \mathscr{Z}$ such that $f(n) \geq g(n)$ for infinitely many $n$, and show that

$$
B=\bigcup\left\{\left[\pi_{i+1}, \pi_{i+2}\right):\left(\exists n \in\left[\pi_{i}, \pi_{i+1}\right)\right) f(n) \geq g(n)\right\} \in \mathscr{B}_{\alpha} .
$$

This completes the proof.

\section{CONSEQUENCES}

An ideal $\mathscr{I}$ is a family of functions closed both downward and under binary maximum; that is the function $h(n)=\max \{f(n), g(n)\}$ belongs to $\mathscr{I}$ whenever $f$ and $g$ do. $\mathscr{I}$ is a growth type if it is an ideal closed under pointwise addition. Since any ideal with the $\mathscr{H}$-property is necessarily dominating, Theorem 7 implies the result of [3] that the minimum number of inequivalent growth types is attained under $\mathfrak{u}<\mathfrak{g}$, namely four.

The result also answers some questions of [6] on the classification of measure zero subsets of $[0,1]$. For $\mathscr{X} \subseteq[0,1]$ of measure zero, we defined (following Borel) a sequence $\left\langle I_{n}: n \in \omega\right\rangle$ of open intervals to be a cover of $\mathscr{Z}$ if

$$
\sum_{n}\left|I_{n}\right|<\infty \text { and } \mathscr{X} \subseteq \bigcap_{n} \bigcup_{k \geq n} I_{k}
$$

which means in particular that each $x \in \mathscr{X}$ must belong to infinitely many $I_{n}$. Now put

$$
f_{I}(n)=\left\lceil 1 / \sum_{k \geq n}\left|I_{k}\right|\right\rceil
$$


and

$$
\mathscr{M}(\mathscr{X})=\left\{f_{I}: I \text { is a cover of } \mathscr{X}\right\}
$$

We studied an ordering on sets of measure zero, which was shown to be equivalent to

$$
\mathscr{X} \preceq \mathscr{Y} \quad \text { iff } \mathscr{M}(\mathscr{X}) \preceq^{1} \mathscr{M}(\mathscr{Y}),
$$

and the induced ordering on equivalence classes. It is shown in [6] that there are at least four classes (namely the $\mathscr{L}, \mathscr{U}, \mathscr{H}$ and $\mathscr{D}$ class), and evidently nothing below the $\mathscr{L}$-class. Theorem 7 shows that those are the only ones assuming $\mathfrak{u}<\mathfrak{g}$, answering questions 1 and 3 of [6].

Many other questions remain, for example can we prove in ZFC that a family $\mathscr{X}$ has the $\mathscr{H}$-property if $\mathscr{X} \succ \mathscr{X}(\mathscr{U})$ for all ultrafilters $\mathscr{U}$, or that $\mathscr{X}$ is bounded if $\mathscr{X}(\mathscr{U}) \succ \mathscr{X}$ for all $\mathscr{U}$ ?

To conclude, we present a curious consequence of the minimum number (five) of equivalence classes of functions being attained. To simplify the statement, call a family $\mathscr{A} \subseteq[\omega]^{\omega}$ closed upward under $\subseteq^{*}$ if $B \in \mathscr{A}$ whenever $A \subseteq \subseteq^{*} B$ for some $A \in \mathscr{A}$.

Theorem 10. Assume there are only five classes of functions. Then for any (nonempty) family $\mathscr{A} \subseteq[\omega]^{\omega}$ which is closed upward under $\subseteq^{*}$, there is a finite-to-one function $f$ such that either

$$
\begin{array}{cl}
\text { or } & f(\mathscr{A})=[\omega]^{\omega} \\
\text { or else } & f(\mathscr{A}) \text { is an ultrafilter } \\
& f(\mathscr{A}) \text { the cofinite filter. }
\end{array}
$$

Equivalently, for any (nonempty) family $\mathscr{A} \subseteq[\omega]^{\omega}$, there is a finite-to-one function $f$ such that either

$$
\begin{array}{cl} 
& f(\mathscr{A}) \text { is dense in }\left\langle[\omega]^{\omega} ; \subseteq^{*}\right\rangle, \\
\text { or } & f(\mathscr{A}) \text { generates an ultrafilter, } \\
\text { or else } & f(\mathscr{A}) \text { consists only of cofinite sets. }
\end{array}
$$

This clearly implies statement (3) of [3], which asserts that for any filter $\mathscr{F}$, there is a finite-to-one function $f$ such that $f(\mathscr{F})$ is either the cofinite filter or an ultrafilter; and hence implies NCF.

Proof of the theorem. Fix a family $\mathscr{A}$ of infinite sets which is closed upward under $\subseteq^{*}$, and consider the family of functions $\mathscr{X}(\mathscr{A})$. It must lie in one of the five classes by hypothesis, but clearly cannot be dominating since $\mathscr{A}$ consists of infinite sets only.

Case 1. Suppose $\mathscr{X}(\mathscr{A})$ is bounded by some $g$. We can assume without loss of generality that $g$ is a strictly increasing function, and hence we get for any $A \in \mathscr{A}$ :

$$
\left(\forall^{\infty} a \in A\right)[\operatorname{next}(A, a+1)<g(a+1)] .
$$

Define $\pi_{0}=0$, and by induction $\pi_{i+1}=g\left(\pi_{i}\right)$. This implies that, for any $A \in \mathscr{A}$,

$$
\left(\forall^{\infty} i\right)\left[A \cap\left[\pi_{i}, \pi_{i+1}\right) \neq \varnothing\right],
$$

and, if we define $f(n)=i$ for $n \in\left[\pi_{i}, \pi_{i+1}\right)$, we conclude that $f(\mathscr{A})$ consists only of cofinite sets. Since $\mathscr{A}$ is assumed to be closed under finite modifications of its members, $f(\mathscr{A})$ must consist of all cofinite sets. 
Case 2. Assume now that $\mathscr{Z}(\mathscr{A})$ has the $\mathscr{H}$-property. We show that $f(\mathscr{A})=$ $[\omega]^{\omega}$ for some finite-to-one function $f$.

Fix an unbounded $h$ which witnesses the $\mathscr{H}$-property of $\mathscr{X}(\mathscr{A})$, which means that

$$
(\forall f)\left[\left(\exists^{\infty} n\right) f(n) \leq h(n) \rightarrow f \in \mathscr{X}(\mathscr{A})\right] .
$$

Define $\pi_{0}=0$ and by induction $\pi_{i+1}$ such that $h\left(\pi_{i+1}\right)>\pi_{i}$. Put $f(n)=i$ if $n \in\left(\pi_{i-1}, \pi_{i}\right]$; and $f(0)=0$. We claim that $f$ works. Indeed, for $Y \subseteq \omega$ infinite, let $X=\left\{\pi_{y}: y \in Y\right\}$. The function $h(\operatorname{next}(X,-)) \in \mathscr{X}(\mathscr{A})$ by the $\mathscr{H}$-property of $\mathscr{X}(\mathscr{A})$ since $h(\operatorname{next}(X, x))=h(x)$ for each $x \in X$. We conclude that $h(\operatorname{next}(X,-)) \leq \operatorname{next}(A,-)$ for some $A \in \mathscr{A}$. But this means that, for each $x \in X$,

$$
A \cap(x, h(\operatorname{next}(X, x+1)))=\varnothing,
$$

or equivalently,

$$
A \subseteq \bigcup_{x \in X}[h(x), x]
$$

This shows that $f(A) \subseteq Y$ and hence $f(\mathscr{A})=[\omega]^{\omega}$. This completes the proof of this case.

Case 3. We now assume that $\mathscr{X}(\mathscr{A})$ is equivalent to $\mathscr{X}(\mathscr{U})$ for some ultrafilter $\mathscr{U}$; we show that $f(\mathscr{A})=f(\mathscr{U})$ for some finite-to-one function $f$.

Lemma 11. Assume that $\mathscr{X}(\mathscr{U}) \preceq \mathscr{X}(\mathscr{A})$, then there is a nondecreasing onto finite-to-one function $f$ such that $f(\mathscr{U}) \subseteq f(\mathscr{A})$. That is

$$
(\forall B \in \mathscr{U})(\exists A \in \mathscr{A})[f(A) \subseteq f(B)] .
$$

Proof. We supply the proof for the $\preceq^{2}$ order and leave the similar proof for the first order to the reader. By hypothesis,

$$
(\exists r)(\forall B \in \mathscr{U})(\exists A \in \mathscr{A})(\forall n)[\operatorname{next}(B, n) \leq \operatorname{next}(A, r(n))] .
$$

Define $\pi_{0}=0$ and by induction $\pi_{n+1}>r\left(\pi_{n}\right)$. For $i<2$, define $X(i)=$ $\bigcup_{n}\left[\pi_{2 n+i}, \pi_{2 n+i+1}\right)$. Since $\mathscr{U}$ is an ultrafilter, it must contain one of the $X(i)$, and for notational simplicity we assume that $X=X(0) \in \mathscr{U}$. Put $f(k)=n$ for $k \in\left[\pi_{2 n}, \pi_{2 n+2}\right)$. Now for $B \in \mathscr{U}$, which we can assume to be a subset of $X$, we can fix $A \in \mathscr{A}$ as above and we claim that $f(A) \subseteq f(B)$. Indeed, if $a \in A$, say $a \in\left[\pi_{2 n}, \pi_{2 n+2}\right)$, then

$$
\begin{aligned}
\operatorname{next}\left(B, \pi_{2 n}\right) & =\operatorname{next}\left(B, \pi_{2 n-1}\right) \leq \operatorname{next}\left(A, r\left(\pi_{2 n-1}\right)\right) \\
& \leq \operatorname{next}\left(A, \pi_{2 n}\right)<\pi_{2 n+2} .
\end{aligned}
$$

Hence $f(a) \in f(B)$ as desired.

Lemma 12. Assume $\mathscr{X}(\mathscr{A}) \preceq \mathscr{X}(\mathscr{U})$. Then there is a nondecreasing onto finiteto-one function $f$ such that $f(\mathscr{A}) \subseteq f(\mathscr{U})$. That is

$$
(\forall A \in \mathscr{A})(\exists B \in \mathscr{U})[f(B) \subseteq f(A)] .
$$

Proof. We supply the proof for the $\preceq^{1}$ ordering and leave the similar proof for the second ordering to the reader. By hypothesis, we have

$$
(\exists r)(\forall A \in \mathscr{A})(\exists B \in \mathscr{U})(\forall n)[\operatorname{next}(A, n) \leq \operatorname{next}(r(B), r(n))] .
$$


Put $\pi_{0}=0$ and by induction define $\pi_{n+1}>r\left(\pi_{n}\right)$. As before, we can assume for notational simplicity that $X=\bigcup_{n}\left[\pi_{2 n}, \pi_{2 n+1}\right) \in \mathscr{U}$. Put $f(k)=n$ for $k \in\left[\pi_{2 n}, \pi_{2 n+2}\right)$. We claim that $f$ works. Fix $A \in \mathscr{A}$, and choose $B \in \mathscr{U}$ as above. We can assume that $B \subseteq X$, and we show that $f(B) \subseteq f(A)$. Fix $b \in B$, say $b \in\left[\pi_{2 n}, \pi_{2 n+1}\right)$; then

$$
\begin{aligned}
\operatorname{next}\left(A, \pi_{2 n}\right) & \leq \operatorname{next}(A, b) \leq \operatorname{next}(r(B), r(b)) \\
& =r(b) \leq r\left(\pi_{2 n+1}\right)<\pi_{2 n+2} .
\end{aligned}
$$

We conclude that $f(b) \in f(A)$ as desired.

Now the following will complete the verification of Case 3.

Lemma 13. Assume that $\mathscr{X}(\mathscr{A}) \succeq \mathscr{X}(\mathscr{U})$ and $f$ is a nondecreasing onto finiteto-one function, then $\mathscr{X}(f(\mathscr{A})) \succeq \mathscr{X}(f(\mathscr{U}))$.

Proof. Assume first that $\mathscr{X}(\mathscr{U}) \preceq^{1} \mathscr{X}(\mathscr{A})$, and hence choose a strictly increasing function $r$ such that

$(\forall B \in \mathscr{U})(\exists A \in \mathscr{A})\left(\forall^{\infty} n\right)[\operatorname{next}(B, n) \leq r(\operatorname{next}(A, n))=\operatorname{next}(r(A), r(n))]$.

Hence, for all but finitely many $n$, if $k$ denotes $\min f^{-1}\{n\}$,

$$
\begin{aligned}
\operatorname{next}(f(B), n) & \leq f(\operatorname{next}(B, k)) \\
& \leq f(\operatorname{next}(r(A), r(k))) \\
& =f(r(\operatorname{next}(A, k))) \\
& \leq f\left(r\left(\max f^{-1}\{\operatorname{next}(f(A), n)\}\right)\right)
\end{aligned}
$$

and we conclude that $\mathscr{X}(f(\mathscr{U})) \preceq^{1} \mathscr{X}(f(\mathscr{A}))$.

Now assume that $\mathscr{X}(\mathscr{U}) \preceq^{2} \mathscr{X}(\mathscr{A})$ and without loss of generality fix a strictly increasing function $r$ such that

$$
f(n)<f(r(n)) \text { for each } n
$$

and

$$
(\forall B \in \mathscr{U})(\exists A \in \mathscr{A})\left(\forall^{\infty} n\right)[\operatorname{next}(B, n) \leq \operatorname{next}(A, r(n))] .
$$

Fixing such $A, B, n$, and letting $k=\min f^{-1}\{n\}$, we get

$$
\begin{aligned}
\operatorname{next}(f(B), n) & \leq f(\operatorname{next}(B, k)) \leq f(\operatorname{next}(A, r(k))) \\
& \leq \operatorname{next}\left(f(A), f\left(r^{2}(k)\right)\right) \\
& =\operatorname{next}\left(f(A), f\left(r^{2}\left(\min f^{-1}\{n\}\right)\right)\right)
\end{aligned}
$$

and hence $\mathscr{Z}(f(\mathscr{U})) \varliminf^{2} \mathscr{Z}(f(\mathscr{A}))$ as desired.

To conclude, since we are assuming that $\mathscr{Z}(\mathscr{A}) \preceq \mathscr{X}(\mathscr{U})$ we can fix by Lemma 12 a finite-to-one function $f_{1}$ such that $f_{1}(\mathscr{A}) \subseteq f_{1}(\mathscr{U})$. But $\mathscr{X}(\mathscr{A}) \succeq$ $\mathscr{X}(\mathscr{U})$ and hence $\mathscr{X}\left(f_{1}(\mathscr{A})\right) \succeq \mathscr{X}\left(f_{1}(\mathscr{U})\right)$ by Lemma 13 . Now by Lemma 11 , there is a finite-to-one function $f_{2}$ such that $f_{2}\left(f_{1}(\mathscr{U})\right) \subseteq f_{2}\left(f_{1}(\mathscr{A})\right)$. This clearly implies that $f_{2}\left(f_{1}(\mathscr{U})\right)=f_{2}\left(f_{1}(\mathscr{A})\right)$ and completes the proof of the theorem. 


\section{REFERENCES}

1. A. Blass, Near coherence of filters, II: Applications to operator ideals, the Stone-Čech remainder of the half-line, order ideals of sequences and slenderness of groups, Trans. Amer. Math. Soc. 300 (1987), 557-581.

2. __ Applications of superperfect forcing and its relatives, Set Theory and its Applications, (J. Steprāns and S. Watson, eds.), Lecture Notes in Math., vol. 1401, Springer-Verlag, 1989.

3. A. Blass and C. Laflamme, Consistency results about filters and the number of inequivalent growth types, J. Symbolic Logic 54 (1989), 50-56.

4. R. Göbel and B. Wald, Wachstumstypen und schlanke Gruppen, Sympos. Math. 23 (1979), 201-239.

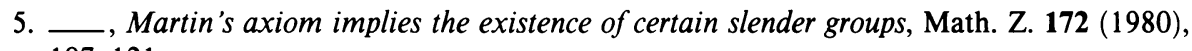
107-121.

6. W. Just and C. Laflamme, Classifying sets of measure zero with respect to their open covers, Trans. Amer. Math. Soc. 321 (1990), 627-645.

7. A. S. Kechris, On a notion of smallness for subsets of the Baire space, Trans. Amer. Math. Soc. 229 (1977), 191-207.

8. J. Saint-Raymond, Approximations des sous-ensembles analytiques par l'intérieur, C.R. Acad. Sci. Paris 281 (1975), A85-A87.

Department of Mathematics, University of Toronto, Toronto, Ontario, Canada M5S$1 \mathrm{~A} 1$

Current address: Department of Mathematics and Statistics, York University, 4700 Keele Street, North York, Ontario, Canada M3J 1P3 